\title{
Double Dissociation between the Anterior Cingulate Cortex and Nucleus Accumbens Core in Encoding the Context versus the Content of Pavlovian Cocaine Cue Extinction
}

\author{
Mary M. Torregrossa, ${ }^{1}$ Jessica Gordon, ${ }^{1}$ and Jane R. Taylor ${ }^{1,2}$ \\ Departments of ${ }^{1}$ Psychiatry and ${ }^{2}$ Psychology, Yale University, New Haven, Connecticut 06508
}

\begin{abstract}
One strategy proposed to treat addictive disorders is to extinguish the association between environmental stimuli (cues) and actions associated with drug use to reduce relapse. The context specificity of extinction learning, however, impairs the ability of addicts to generalize extinction training to the drug-taking context. We previously reported that the NMDA receptor partial agonist D-cycloserine administered after pavlovian extinction of cocaine cues in the nucleus accumbens core (NAc) reduced cue-induced renewal. Nevertheless, it was unclear whether this was due to disrupted contextual encoding of extinction or enhanced extinction consolidation. Thus, we examined the effect of the NMDA receptor antagonist D-AP5 on context encoding versus cue extinction learning. We also determined the role of the anterior cingulate cortex (ACC) in encoding the cue extinction memory or the context, due to its projections to NAc, and hypothesized the role in conflict monitoring and contextual modulation of decision making. Using rats, we observed that NMDA receptor antagonism in the NAc did not alter context encoding but did interfere with acquisition of the cue extinction memory, i.e., learning, conversely inactivation of the ACC reduced the contextual encoding of extinction but did not interfere with the acquisition or expression of extinction. The observed effects were not present in the absence of cue extinction training. Additionally, the contextual memory did not appear to be consolidated in the ACC as neither postsession inactivation nor protein synthesis inhibition impaired context-appropriate responding. These results have implications for overcoming the context specificity of extinction to treat psychiatric disorders including addiction.
\end{abstract}

\section{Introduction}

Few effective treatments for addictive disorders exist. Recent research has focused on combining behavioral and pharmacological interventions as alternative therapies. For example, using pharmacological "cognitive enhancers" to facilitate the extinction of drug-associated memories (Taylor et al., 2009; Torregrossa and Taylor, 2013). Extinction therapy, however, has generally been ineffective in treating addiction. This ineffectiveness is likely due to the context specificity of extinction learning (Bouton et al., 2006). Thus, when one returns to the drug-taking context after extinction in a treatment center context, a form of relapse is induced, termed renewal (Bouton et al., 2012). We previously reported that merging pavlovian extinction of a cocaineassociated cue with postsession intra-nucleus accumbens core (NAc) infusions of the NMDA receptor (NMDAR) partial coagonist, D-cycloserine (DCS), potently reduced renewal suggest-

Received Feb. 1, 2013; revised April 5, 2013; accepted April 5, 2013.

Author contributions: M.M.T. and J.R.T. designed research; M.M.T. and J.G. performed research; M.M.T. analyzed data; M.M.T. and J.R.T. wrote the paper.

This work was supported by United States Public Health Service Grants K01DA031745 and DA015222. The authors declare no competing financial interests.

Correspondence should be addressed to Mary M. Torregrossa, Bridgeside Point II, 450 Technology Drive, Pittsburgh, PA 15219. E-mail: torregrossam@upmc.edu.

M.M. Torregrossa's present address: University of Pittsburgh, Bridgeside Point II, 450 Technology Drive, Pittsburgh, PA 15219.

DOI:10.1523/JNEUROSCI.0489-13.2013

Copyright $\odot 2013$ the authors $\quad 0270-6474 / 13 / 338370-08 \$ 15.00 / 0$ ing that cue extinction could be enhanced to overcome context specificity (Torregrossa et al., 2010). Whether DCS enhanced consolidation of the cue extinction memory, making it stronger and more generalizable, or if DCS interfered with context encoding, such that the extinction memory was expressed independent of context, remain to be determined. Indeed, morphine in the NAc has been shown to interfere with appropriate contextual encoding of fear extinction (Westbrook et al., 1997). Because DCS is a partial agonist it could block contextual learning through interference with glycine-mediated signaling, or facilitate cue extinction memory consolidation by enhancing NMDAR signaling. Therefore, we determined whether antagonizing NMDARs in the NAc interferes with context-appropriate expression of cue extinction versus cue extinction learning itself.

Additionally we investigated the neuroanatomical locus for encoding context during cue extinction learning. Studies using conditioned fear identified the hippocampus, entorhinal cortex, and fornix in the contextual modulation of extinction retrieval (Corcoran et al., 2005; Hobin et al., 2006; Ji and Maren, 2005, 2008a,b). However, there is little evidence for sites required for acquiring contextual information during extinction that are not involved in extinction learning itself. One candidate region is the anterior cingulate cortex (ACC). The ACC projects to the entorhinal cortex, which then sends information to the hippocampus, and due to the requirement of the entorhinal cortex for contextappropriate behavior after extinction of conditioned fear (Ji and Maren, 2008b), it is a cortical region likely involved in encoding 
contextual information. Moreover, the ACC is a region that monitors conflict, responds to prediction error, and evaluates the value of expected rewards (Botvinick, 2007; Totah et al., 2009; Kennerley et al., 2011; Rushworth et al., 2011; Roesch et al., 2012). These processes all occur during extinction, where the expected reward is not received after cue presentation or performance of an action (Taylor et al., 2009; Torregrossa and Taylor, 2013). The ACC is also thought to be involved in action selection based on the current state of the environment (Walton and Mars, 2007), and thus would be hypothesized to be engaged in monitoring the context during a case of unexpected lack of reward, as occurs in extinction. Therefore, we determined the consequence of pharmacological inactivation of the ACC, to determine its absolute role in encoding or storing contextual information during extinction.

\section{Materials and Methods}

Animals. Experimental subjects were male Sprague Dawley rats weighing 275-325 g upon delivery (Charles River). Rats were housed in a temperature- and humidity-controlled room on a $12 \mathrm{~h} \mathrm{light/dark} \mathrm{cycle}$ with ad libitum access to food and water except for periods of food restriction described below. Animal procedures conformed to the policies of the Yale University Institutional Animal Care and Use Committee and the National Institutes of Health Guidelines on the Care and Use of Laboratory Animals.

Drugs. Cocaine hydrochloride (provided by National Institute on Drug Abuse, Research Triangle Park, NC) was dissolved in sterile $0.9 \%$ saline and filtered for self-administration. Baclofen, muscimol, and $\mathrm{D}(-)$-2-amino-5-phosphonovaleric acid (D-AP5) (Sigma-Aldrich) were prepared in sterile $0.9 \%$ saline. Cycloheximide (CHX; Sigma-Aldrich) stock solution was dissolved in 100\% dimethylsulfoxide (DMSO). The infusion concentration was achieved by diluting with double distilled water. The concentration of DMSO infused was $20 \%$.

Surgical procedures. Rats were anesthetized with a combination of 87.5 $\mathrm{mg} / \mathrm{kg}$ ketamine and $5 \mathrm{mg} / \mathrm{kg}$ xylazine. Rats were administered $5 \mathrm{ml}$ of lactated Ringer's solution and $5 \mathrm{mg} / \mathrm{kg}$ of the analgesic Rimadyl before implantation with indwelling jugular catheters (CamCaths) as described previously (Torregrossa and Kalivas, 2008; Torregrossa et al., 2010). In addition, intracranial cannulae (22 gauge; Plastics One) were implanted bilaterally dorsal to the brain region of interest using the following coordinates according to the atlas of Paxinos and Watson (2005) given in millimeters with reference to bregma: NAc anteroposterior (AP): +1.6 , mediolateral (ML): \pm 1.5 , dorsoventral (DV): -6.0 ; ACC AP: +2.5 , ML: $\pm 0.75, \mathrm{DV}:-1.8$. Obturators were inserted in the guide cannulae to maintain patency.

Cocaine self-administration. Rats were trained to self-administer cocaine using standard procedures (Torregrossa and Kalivas, 2008; Torregrossa et al., 2010). Rats were maintained at $\sim 90 \%$ of their free-feeding body weight throughout the experiments. Training was conducted in standard operant conditioning chambers (Med Associates), containing a metal grid floor, two retractable levers located on one wall of the chamber, a tone-generator, stimulus-light, and infusion pump. One lever (counterbalanced across rats) was designated the active lever and the opposite lever the inactive lever. Rats were allowed to self-administer $1.0 \mathrm{mg} / \mathrm{kg} /$ infusion cocaine during daily $1 \mathrm{~h}$ sessions. Sessions were automatically terminated after $60 \mathrm{infu}-$ sions to avoid overdose. Responses on the active lever were reinforced on a fixed ratio 1 (FR1) schedule of reinforcement with a $10 \mathrm{~s}$ timeout. Each cocaine infusion was paired with a $10 \mathrm{~s}$ tone-light cue. Inactive lever responses had no programmed consequences. Rats were trained for at least $10 \mathrm{~d}$ and until they self-administered at least 8 infusions/d over 2 consecutive days. Group numbers for each experiment are listed in the figure captions.

Lever extinction. After self-administration training, lever extinction was conducted as previously reported (Torregrossa et al., 2010) and consisted of daily $1 \mathrm{~h}$ sessions where lever presses had no programmed consequences. Lever extinction continued for at least $7 \mathrm{~d}$ and until the number of lever presses was reduced to $<25$ lever presses on the last 2 consecutive days of extinction.

Pavlovian cue extinction. Following lever extinction, groups underwent $2 \mathrm{~d}$ of pavlovian cue extinction (Torregrossa et al., 2010). During pavlovian extinction, animals were placed into novel operant conditioning chambers that were distinct from the self-administration chambers, differing in size, flooring texture, shape, and smell (Context B). During pavlovian extinction there was no opportunity for instrumental responding, i.e., the levers were retracted and the nosepoke holes were occluded. The pavlovian extinction sessions consisted of 30 min sessions where the $10 \mathrm{~s}$ tone-light cue that had been paired with cocaine delivery during self-administration was presented $2 \times / \mathrm{min}$ for a total of 60 -cue presentations per day. In most experiments, a no extinction control group (No Ext) was included to control for placement in a novel box, handling, and any nonspecific or long-lasting effects of the experimental manipulations. These animals were treated exactly the same as the cue extinction groups (Cue Ext), but received no cue presentations during the sessions. Either immediately before or immediately following both of these pavlovian cue extinction or no extinction sessions, experimental compounds or vehicle solutions were administered directly into the brain region of interest (see methodological description below). Presession infusions examined the necessity of a brain region for acquisition of extinction learning and/or contextual learning, while postsession infusions examined the relevance of the region or signaling cascade for consolidation of the extinction and/or contextual memory. All tests described below assess the expression of extinction, but because all of the manipulations were conducted on extinction days and not on test days, we presume that the manipulations are affecting acquisition/consolidation of extinction or contextual memories and not the retrieval or expression of memories.

Cue-induced reinstatement (test for contextual encoding). Cue-induced reinstatement was conducted in the original self-administration/drugtaking context (Context $\mathrm{A}$ ), because context-appropriate renewal must be tested in a context other than the cue extinction context where original learning occurred. The day after pavlovian cue extinction, cue-induced reinstatement was assessed during a $1 \mathrm{~h}$ session where active lever presses produced the $10 \mathrm{~s}$ cocaine-associated cue on an FR1 schedule, but no other reinforcement. Inactive lever presses were monitored but had no programmed consequences. Due to the context specificity of extinction, it was expected that vehicle infused animals would display as much reinstatement in Context A as No Ext controls, but that if the contextual information was not acquired or consolidated during pavlovian extinction in Context B, a reduction in reinstatement would be observed, as the extinction learning would generalize across contexts and reduce renewal.

Test of conditioned reinforcement (test for extinction learning). The day after cue reinstatement testing, the ability of the cocaine-associated cue to support acquisition of a new response, known as conditioned reinforcement, was assessed in Context B. Preliminary data indicated that the pavlovian extinction procedure is sufficient to reduce responding for a cocaine-conditioned cue relative to no extinction controls when the extinction training and testing occur in the same context. Thus, the conditioned reinforcement test in Context $\mathrm{B}$ serves as a measure of the successful acquisition, consolidation, and expression of extinction learning. To test for the effectiveness of extinction learning and expression the conditioned reinforcement test had to be conducted in Context $B$, where the cue extinction training occurred. Conditioned reinforcement tests consisted of a $1 \mathrm{~h}$ session where rats learned to make a new response (nosepoke) that was solely reinforced by presentation of the $10 \mathrm{~s}$ tonelight cue associated with cocaine self-administration. Active nosepokes produced the cue on an FR1 schedule. Inactive nosepokes were recorded but produced no programmed consequences, and a significantly greater number of active over inactive responses indicates that the cue has conditioned reinforcing properties (Taylor and Robbins, 1984, 1986). Inactive nosepokes were only counted after the first correct, active nosepoke to avoid including any side biases in the analysis of whether the cue could support acquisition of a new response for conditioned reinforcement over nonspecific responses (Olausson et al., 2004); however, it should be noted that active responses could also be influenced by side biases. In addition, animals that made no responses during this test were not in- 
a

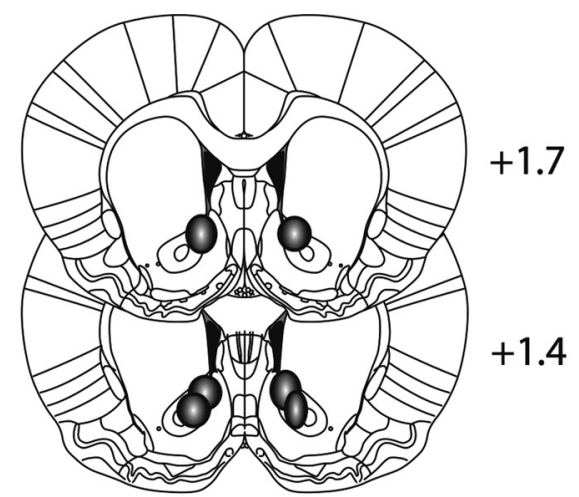

C

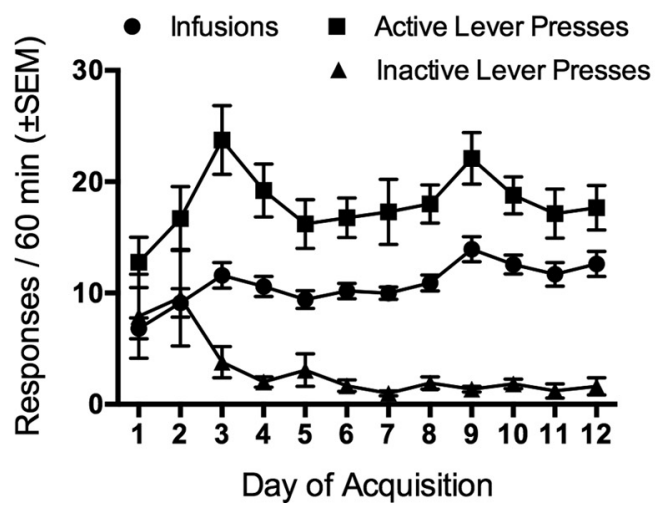

b

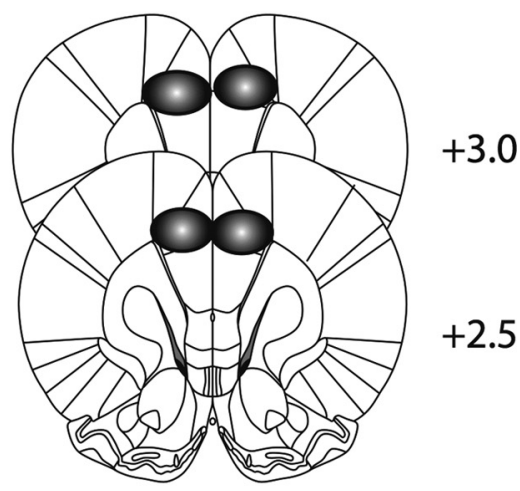

d

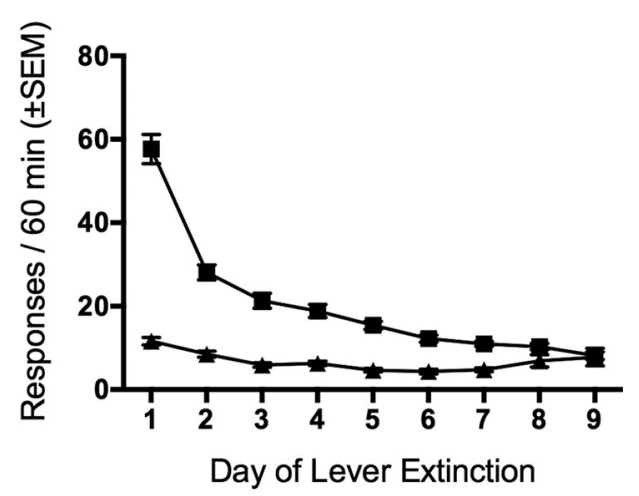

Figure 1. Histological representation of infusion locations and pretreatment training. $\boldsymbol{a}$, Location of range of infusions in the NAc. $\boldsymbol{b}$, Location of range of infusions into the ACC. Numbers by each figure represent millimeters from bregma. c, Cocaine self-administration training across days averaged across all experimental groups illustrating active and inactive lever presses and infusions earned. $\boldsymbol{d}$, Lever extinction training across days averaged across all experimental groups illustrating active and inactive lever presses.

cluded in these analyses as they never had the opportunity to learn that nosepoke responses produced cue presentations. A total of seven animals in all of the experiments were removed from the conditioned reinforcement analyses due to failure to respond.

Drug infusions. Infusions were given by removing obturators and inserting injection cannulae (28 gauge; Plastics One) that extended $1 \mathrm{~mm}$ beyond the guide cannulae. The injectors were connected to Hamilton syringes controlled by a syringe pump via polyethylene tubing. Infusions were given in a volume of $0.3 \mu \mathrm{l}$ in the ACC and $0.5 \mu \mathrm{l}$ in the NAc over 2 $\mathrm{min}$, and injectors were left in the cannulae for an additional $1 \mathrm{~min}$ to allow for drug diffusion. A combination of baclofen and muscimol (Bac/ Mus) was infused at a dose of $0.3 / 0.03 \mathrm{nmol} / \mathrm{side}, \mathrm{D}-\mathrm{AP} 5$ was infused at a dose $2 \mu \mathrm{g}$ per side, and CHX was infused at a dose of $20 \mu \mathrm{g} / \mathrm{side}$. Control groups received the same volume infusion of the appropriate vehicle solution.

Statistical analysis. Data were analyzed using GraphPad Prism version $5.0 \mathrm{~b}$ for Macintosh (GraphPad Software). Reinstatement tests were analyzed by two-way ANOVA with repeated measures with the withinsubjects factor being responding on the last day of lever extinction versus reinstatement responding and the between-subjects factor being the cue extinction treatment. For the conditioned reinforcement tests, the between-subjects factor was cue extinction treatment while the withinsubjects factor was active versus inactive nosepoke. Significant effects were further analyzed by Bonferroni's post hoc $t$ tests, with significance set at $p<0.05$.

\section{Results}

\section{Histology}

Figure 1, $a$ and $b$, illustrates the location of placements in the NAc and ACC, respectively, for all of the experiments. Any animals with placements outside of the targeted region or with substantial cannula damage to the ACC were excluded from the analyses. A total of five animals with inaccurate placement/damage in the ACC and one animal with inaccurate placement in NAc were removed from the study.

\section{Self-administration and lever extinction training}

Figure 1, $c$ and $d$, illustrates the self-administration acquisition curve and lever extinction curve, respectively, for all animals used in the experiments. There were no statistical differences between any of the treatment groups during these phases of the experiment before any treatments being given. The statistical details for each experiment are listed in the sections below.

\section{Effect of NMDAR antagonism in the NAc on contextual encoding versus extinction learning}

In the first experiment, we examined whether the NMDAR antagonist, AP-5, infused into the NAc before pavlovian extinction training would alter contextual encoding of extinction or extinction learning itself. There were no statistical differences between any of the "to-be" treatment groups on infusions earned across days of self-administration $\left(F_{(3,396)}=0.25, p>0.05\right)$, or on the rate of active lever extinction $\left(F_{(3,308)}=0.16, p>0.05\right)$. Subsequently animals underwent cue/no cue extinction and treatment and were tested. The experimental time line is illustrated in Figure $2 a$. First, animals were tested for renewal of cue-motivated cocaine seeking in a cue-induced reinstatement test in Context A. Animals that received infusions of AP- 5 before pavlovian extinction training had equivalent levels of renewal as compared with vehicle-infused animals (Fig. 2b). Analysis of the data by two-way ANOVA with repeated measures (rmANOVA) indicated a signif- 
a

Intra-NAc Infusion

Training/Testing:

Context:

\begin{abstract}
Cocaine $\rightarrow$ Self-Administration
\end{abstract}

A

b

Pre-Session Infusion Context A - Renewal Test

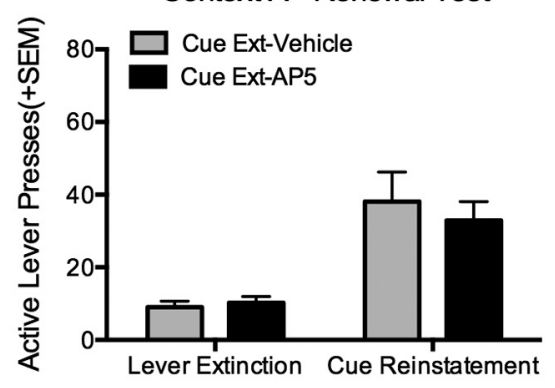

d Context B - Extinction Retrieval Test

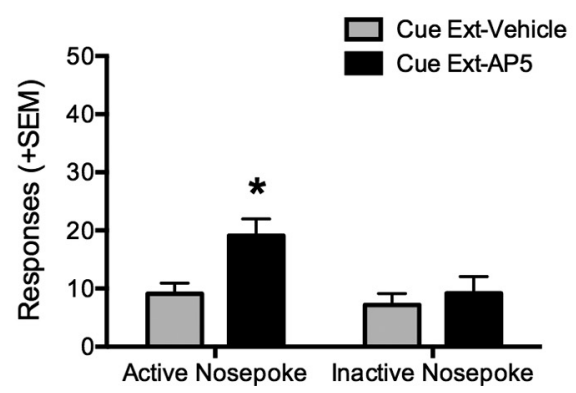

C

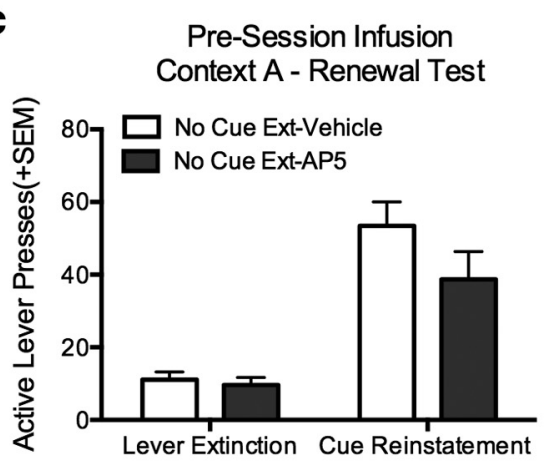

e

Context B - Extinction Retrieval Test

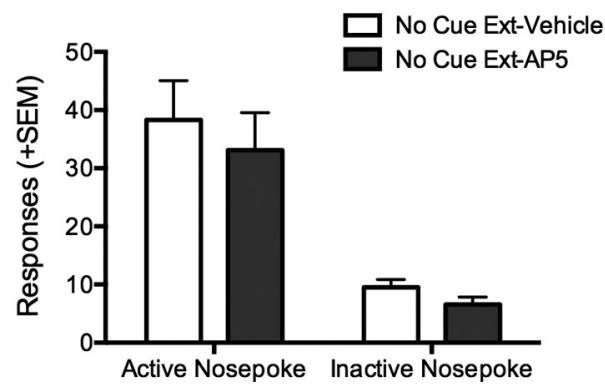

Figure 2. NMDAR blockade in the NAc inhibits cue extinction learning. $\boldsymbol{a}$, Experimental time line. $\boldsymbol{b}$, NMDAR blockade in the NAc with D-AP5 before cue extinction training in Context B did not prevent renewal of cue-induced cocaine seeking in Context A. $N=12 /$ group. $c$, There was also no effect of NMDAR blockade on the renewal of cocaine seeking in the absence of cue extinction training. $N=13$, vehicle; $N=11$ D-AP5. $\boldsymbol{d}$, In a test of extinction recall/expression in the extinction Context B, animals receiving intra-NAC D-AP5 showed significantly more responding for conditioned reinforcement than controls, indicating less expression of cue extinction, ${ }^{*} p<0.05 . N=12$ (vehicle) and 11 (AP-5).e, NMDAR antagonism had no effect on responding for conditioned reinforcement in the absence of cue extinction training (№ Cue Ext). $N=12$ (vehicle) and 11 (AP-5).

icant effect of test phase (lever extinction vs reinstatement) $\left(F_{(1,22)}=37.20, p<0.001\right)$, but no effect of treatment $\left(F_{(1,22)}=\right.$ $0.12, p>0.05$, or interaction $\left(F_{(1,22)}=0.57, p>0.05\right)$. The No Ext groups also showed an equivalent amount of cue-induced reinstatement with a significant effect of test phase $\left(F_{(1,22)}=\right.$ $44.81, p<0.001)$, but no effect of treatment $\left(F_{(1,22)}=2.49, p>\right.$ 0.05 ), or interaction $\left(F_{(1,22)}=1.55, p>0.05\right)$ (Fig. 2c). Therefore, there was no effect of intra-NAc AP-5 given in conjunction with pavlovian cue extinction, or not, on cue-induced renewal/reinstatement of cocaine seeking, indicating no effect on contextual encoding (i.e., context appropriate renewal).

On the other hand, when the animals were tested for expression/retention of cue extinction learning in a conditioned reinforcement test in Context B, we did observe an effect of intra-NAc NMDAR blockade. First, there was an overall significant effect of the cue extinction training procedure to reduce responding for conditioned reinforcement compared with the no extinction groups (significant interaction between condition and active vs inactive nosepoke $\left.\left[F_{(1,43)}=22.88, p<0.001\right]\right)$ indicating that the pavlovian extinction procedure is effective in reducing cuemotivated behavior. Analysis of each condition by rmANOVA indicated that in the group undergoing cue extinction, there was a significant effect of active versus inactive nosepoke $\left(F_{(1,21)}=\right.$ $20.86, p<0.001)$, no effect of treatment $\left(F_{(1,21)}=3.36, p>0.05\right)$, but, importantly, a significant interaction between treatment and nosepoke $\left(F_{(1,21)}=9.57, p=0.006\right)$. Bonferroni's post hoc analysis indicated that the AP-5-treated group responded significantly more on the active nosepoke than the control group, whereas there was no difference in inactive nosepoke responding (Fig. $2 d$ ). On the other hand, in the absence of pavlovian cue extinction training, both vehicle and AP-5-treated groups showed high levels of responding for conditioned reinforcement with a significant effect of nosepoke $\left(F_{(1,21)}=43.23, p<0.001\right)$, but no effect of treatment $\left(F_{(1,21)}=1.56, p>0.05\right)$, or interaction $\left(F_{(1,21)}=0.07, p>0.05\right)$ (Fig. 2e). Therefore, the AP-5 infused group likely had a partial impairment in cue extinction learning/ expression, but not a long-term, extinction independent, effect on performance or motivation that confounds interpretation of the results.

\section{Effect of inactivation of the ACC on contextual encoding} versus extinction learning

Next, we examined whether activity in the ACC is required to encode the context during pavlovian cue extinction or acquisition of cue extinction learning itself. We used Bac/Mus to activate GABAB and GABAA receptors in the ACC, producing a reversible inactivation of the region during pavlovian cue extinction training. There were no statistical differences between any of the 

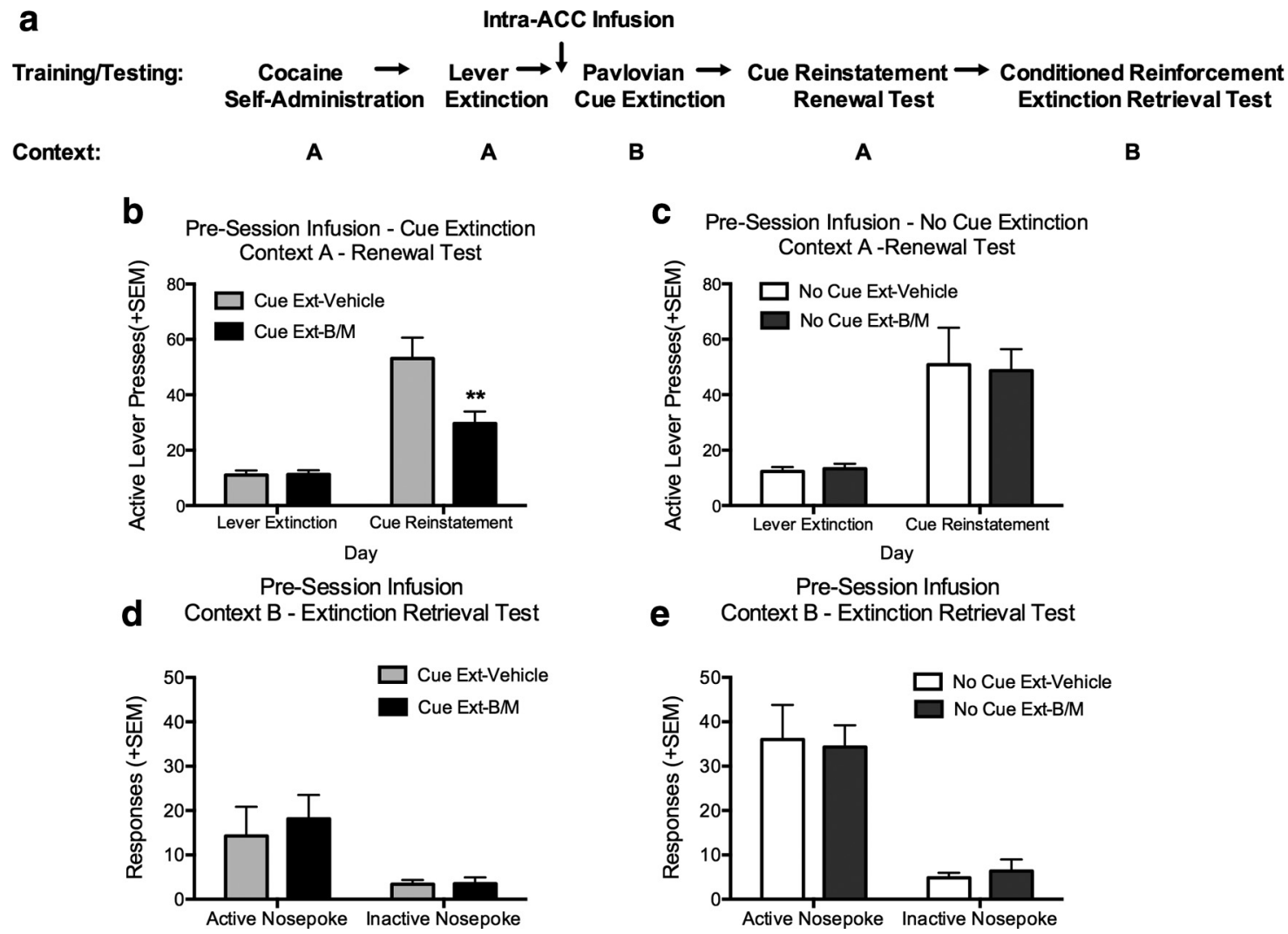

Figure 3. ACC activity is required to encode the context during cue extinction. $\boldsymbol{a}$, Experimental time line. $\boldsymbol{b}$, Inactivation of the $A C C$ with Bac/Mus (B/M) before cue extinction training in Context $B$ inhibited renewal of cue-induced cocaine seeking in Context $A,{ }^{* *} p<0.01, N=8 /$ group. $c$, Prior intra-ACC B/M had no effect on cue-induced renewal in the absence of cue extinction training. $N=8$, vehicle; $N=6, B / M . d$, Intra-ACC B/M during cue extinction had no effect on subsequent expression of cue extinction in a test for conditioned reinforcement in Context $B . N=8$ (vehicle); $N=6(B / M) . e$, Prior intra-ACC B/M also had no effect on the expression of conditioned reinforcement in the absence of cue extinction training. $N=7$ (vehicle); $N=6(B / M)$.

to-be treatment groups on infusions earned across days of selfadministration $\left(F_{(3,288)}=2.27, p>0.05\right)$, or on the rate of active lever extinction $\left(F_{(3,203)}=1.58, p>0.05\right)$. Subsequently animals underwent cue/no cue extinction and treatment and were tested. The experimental time line is illustrated in Figure $3 a$. First, we tested for context-appropriate renewal of cocaine cue-induced responding in Context A. Analysis by rmANOVA indicated a significant effect of test phase $\left(F_{(1,14)}=40.60, p<0.001\right)$, treatment $\left(F_{(1,14)}=7.62, p=0.015\right)$, and interaction $\left(F_{(1,14)}=6.26\right.$, $p=0.025)$. Post hoc analyses revealed that the Bac/Mus-treated group had significantly less cue-induced renewal than the controls, while there was no effect before treatment on responses on the last lever extinction day (Fig. 3b). Moreover, while there was a significant effect of test phase for the No Ext groups $\left(F_{(1,14)}=\right.$ $30.70, p<0.001)$, there was no effect of treatment $\left(F_{(1,14)}=\right.$ $0.006, p>0.05)$, or interaction $\left(F_{(1,14)}=0.05, p>0.05\right)$, indicating that in the absence of pavlovian extinction training all animals showed significant cue-induced reinstatement in Context $\mathrm{A}$, and that there was no nonspecific effect of intraACC Bac/Mus infusion on motivation or performance in this test (Fig. 3c).

In addition, while the results of the renewal test strongly indicate that ACC activity was necessary for contextual encoding, but not for acquiring pavlovian extinction, we did test whether all groups showed context-appropriate expression of cue extinction learning using a conditioned reinforcement test in Context B. The No Ext groups were expected to respond much more for conditioned reinforcement, as they never experienced cue extinction in this context. Indeed, while there was a significant effect of test phase for both the Cue $\operatorname{Ext}\left(F_{(1,12)}=8.78, p=0.011\right)$ and No Ext $\left(F_{(1,10)}=51.06, p<0.001\right)$ groups, the mean number of active responses for the No Ext groups was significantly greater than the Cue Ext groups, indicating the effectiveness of the pavlovian cue extinction procedure (significant interaction $\left[F_{(1,25)}=\right.$ $9.95, p=0.004])$. In addition, there was no significant effect of $\mathrm{Bac} / \mathrm{Mus}$ treatment or interaction effect in either the Cue Ext or No Ext condition $\left(F_{(1,12)}=0.17, p>0.05 ; F_{(1,12)}=0.19, p>0.05\right.$; $\left.F_{(1,10)}=0.0002, p>0.05 ; F_{(1,10)}=0.15, p>0.05\right)$. Therefore, intra-ACC Bac/Mus did not affect the acquisition or expression of cue extinction learning as measured by the ability to respond for conditioned reinforcement (Fig. 3d,e), only the ability to exhibit context appropriate renewal.

Effect of postsession ACC inactivation or protein synthesis inhibition on contextual encoding

Finally, we wanted to determine whether the ACC was only necessary for acquiring contextual information at the time of new learning or if the contextual memory was consolidated and stored in the ACC. To test this, we allowed the ACC to be on-line during the pavlovian cue extinction phase and only inactivated the region at the end of the cue extinction sessions. As an additional test for memory consolidation, we similarly infused the protein synthesis inhibitor, CHX, into the ACC after the cue extinction sessions. In this experiment, there was a statistical difference between the "to-be" treatment groups on infusions earned across days of self-administration $\left(F_{(2,310)}=29.58, p<0.001\right)$ and a significant interaction with day of self-administration $\left(F_{(18,310)}=\right.$ $1.92, p<0.05)$. However, post hoc tests indicated that the only 

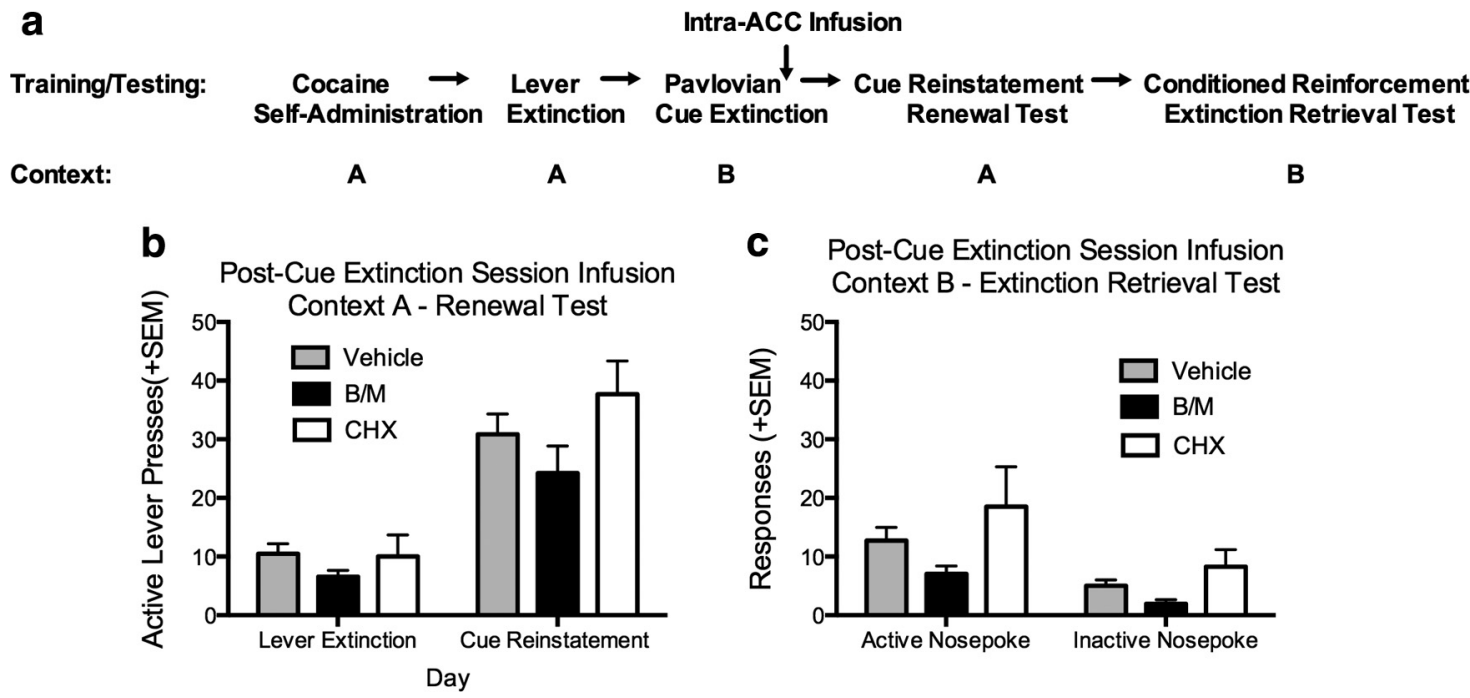

C Post-Cue Extinction Session Infusion Context B - Extinction Retrieval Test

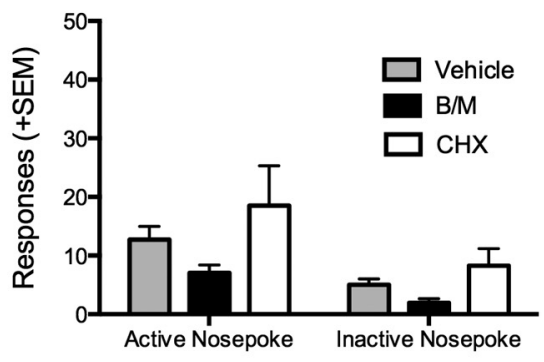

Figure 4. Contextual memory is not consolidated in the ACC. $\boldsymbol{a}$, Experimental time line. $\boldsymbol{b}$, Post cue extinction session administration of Bac/Mus (B/M) or CHX into the ACC had no effect on the expression of context-appropriate renewal of cue-induced cocaine seeking in Context A. $N=19$, vehicle (collapsed groups); $N=9, B / M ; N=6, C H X$. c, Postsession infusion of B/M and CHX in ACC also had no effect on the expression of cue extinction memory in Context B, indicating that neither contextual nor cocaine cue extinction memory is stored in the $A C C . N=18$ (vehicle); $N=9(B / M)$; $N=4(\mathrm{CHX})$.

significant difference between groups was on day 2 of selfadministration where the to-be Bac/Mus group self-administered significantly more infusions than the other two groups. This appeared to be an anomaly because no other day of selfadministration showed a significant difference. In addition, there were no significant differences in the rate of active lever extinction $\left(F_{(2,217)}=2.69, p>0.05\right)$. Subsequently animals underwent cue/no cue extinction and treatment and were tested. The experimental time line is illustrated in Figure $4 a$. There were no significant differences in behavior based on the vehicle infused for each drug, so the two vehicle groups were combined, and both the $\mathrm{Bac} / \mathrm{Mus}$ and CHX data were analyzed and plotted together. Analysis of the cue-induced renewal test of context-appropriate responding revealed a significant effect of test phase $\left(F_{(1,31)}=\right.$ 92.01, $p<0.001)$, but no effect of treatment $\left(F_{(2,31)}=1.50\right.$, $p>0.05)$, or significant interaction $\left(F_{(2,31)}=1.32, p>0.05\right)$. Therefore, neither postsession infusion of Bac/Mus nor CHX affected consolidation of the contextual memory associated with cue extinction (Fig. 4b). Analysis of the conditioned reinforcement test of expression of cue extinction indicated a significant effect of nosepoke $\left(F_{(1,28)}=24.27, p<0.001\right)$, and treatment $\left(F_{(2,28)}=3.50, p=0.044\right)$, but no interaction $\left(F_{(2,28)}=0.77, p>\right.$ $0.05)$. Inspection of the data reveals that while the Bac/Mus group responded less than the other groups, responding was lower on both the inactive and active nosepokes, indicating no real difference in the conditioned reinforcing effects of the cocaine cue (Fig. $4 c$ ). All groups did respond at low levels on the test for conditioned reinforcement as is expected after pavlovian cue extinction training, and no group appeared to have any disruption in the consolidation of the cue extinction memory.

\section{Discussion}

The present study examined the brain regions underlying pavlovian cue extinction learning versus that which encodes the context associated with the cue extinction learning. We found that NMDAR antagonism in the NAc at the time of pavlovian cue extinction training produced a subsequent increase in responding for conditioned reinforcement consistent with partial impairment in the learning/consolidation of the cue ex- tinction memory. However, it should be noted that the observed differences in conditioned reinforcement are possibly skewed by inactive nosepoke responding before the first active, reinforced nosepoke that were not included in our data analysis.

In contrast, we found that inactivation of the ACC during cue extinction training prevented context appropriate expression of cue extinction learning when the animals were tested for renewal outside the extinction context. These results represent a double dissociation that implicates the ACC in the on-line encoding of contextual information during cue extinction, but not in encoding the cue extinction memory itself; whereas, the NAc is necessary for pavlovian extinction learning. Moreover, while the ACC needs to be on-line during cue extinction to encode the context, the contextual memory itself does not appear to be stored and consolidated in the ACC, as postsession inactivation or protein synthesis inhibition did not alter context-appropriate responding. The results of the present studies have important implications for developing extinction-based addiction therapies that overcome the context dependency of extinction to reduce relapse (Crombag et al., 2008; Torregrossa and Taylor, 2013). However, due to the configuration of our operant equipment, we were not able to counterbalance self-administration and pavlovian extinction. Therefore, the results should be interpreted with the understanding that there is a possibility, though we think it is unlikely, that context $\times$ test type $\times$ treatment interactions exist that could influence our results.

We have previously reported that DCS given both systemically and directly into the NAc after pavlovian extinction can reduce subsequent cocaine cue-induced renewal of drug seeking (Torregrossa et al., 2010). This remains one of the few studies identifying a mechanism for overcoming the context dependency of extinction to improve the effectiveness of extinction therapy in the clinical setting. Unfortunately, clinical studies attempting to use DCS to facilitate extinction therapy to treat addiction have had little success (Watson et al., 2011; Price et al., 2013). Surprisingly, however, few of these studies have actually examined the effectiveness of DCS plus extinction training on outcomes outside of the laboratory (Myers and Carlezon, 2012). Therefore, it 
still remains to be seen whether DCS might inhibit renewal in human substance abusing populations, though it should be noted that DCS inhibits renewal of fear in phobic individuals (Ressler et al., 2004). The results presented here strongly suggest that postsession DCS, as reported in Torregrossa et al. (2010), does enhance cue-extinction memory and generalizability across contexts, rather than affecting contextual encoding or other process.

The present study indicates that the contextual encoding of cue extinction and cue-extinction learning itself are dissociable processes, involving distinct neural circuits, allowing the possibility that they can be modulated independently and simultaneously to further improve the effectiveness of extinction therapy. The present study is one of the first to definitively implicate the ACC in context encoding during new learning. A multitude of previous studies have suggested that the ACC is important for accurate decision making based on the task context (Walton and Mars, 2007; Walton et al., 2007; Rushworth et al., 2011), but no previous studies have specifically determined whether the ACC is necessary for context encoding during extinction. Extinction learning provides an excellent means for understanding context encoding because of the clear association between context and expression of extinction (Bouton, 2004; Bouton et al., 2006). While no previous studies have directly assessed the role of ACC in encoding the context specificity of extinction, a recent paper has reported that low-frequency stimulation of the ACC, which inhibits neural activity, in combination with extinction training, prevents the spontaneous recovery of fear (Klavir et al., 2012). Notably, the design of the study by Klavir and et al. (2012) could be interpreted as having altered contexts, which may have made the activity in ACC necessary for appropriate fear expression. On the other hand, it is possible that inactivating the ACC during extinction training simply allows for more effective extinction learning to occur, possibly by removing a part of the circuit that maintains the original conditioned memory. However, in most cases facilitating extinction does not result in a reduction in renewal (cf., Peters et al., 2010), and we would have expected to observe a reduction in the expression of extinction in the conditioned reinforcement test, which we did not.

Further evidence supporting a role for the ACC in context encoding during extinction comes from a human neuroimaging study examining anxiety-provoking context conditioning. In this study, the dorsal ACC (dACC) was activated by extinction of a conditioned context, and the AACC showed significant connectivity with the hippocampus and the amygdala. We postulate that the ACC may provide context-specific information to the hippocampus and extinction information to the amygdala (Lang et al., 2009). Fewer imaging studies of extinction of drug-associated cues or contexts have been conducted, but it is possible that extinction of drug-associated cues also engages an ACC-hippocampus network to encode context, while the ventral striatum (possibly in conjunction with the amygdala and/or ventromedial prefrontal cortex) is necessary to encode extinction learning. Our data would suggest that ACC activation is necessary to acquire context-specific information and that the connectivity to hippocampus is likely responsible for consolidating and storing context-specific information, as postsession disruption of the ACC did not prevent context-appropriate behaviors and previous studies implicate the hippocampus in expressing contextual information (Corcoran et al., 2005; Ji and Maren, 2005; Hobin et al., 2006). Moreover, a recent study in nonhuman primates found that the ACC is particularly engaged in the presence of discrete cues associated with cocaine self-administration (Baeg et al., 2009). While this study did not assess ACC activation during early extinction, it does suggest that the ACC would be very responsive during cocaine cue presentations early in extinction, especially due to the prediction error created in early extinction, where cues are presented without the expected reward. The ACC may be particularly concerned with attending to and monitoring the context of this negative prediction error for future decision making (Bryden et al., 2011).

Previous studies have found that the NAc undergoes significant synaptic plasticity, particularly after extinction training, that may be important for acquiring and maintaining the extinction memory to reduce later reinstatement when the context remains the same (Knackstedt et al., 2010). Overall, the present studies support a role for the NAc in acquiring extinction of cocaineassociated cues, as has been suggested by previous work, and extends these findings to suggest that this may be context dependent (Torregrossa et al., 2010). Moreover, this is the first study to implicate the ACC in encoding contextual information during extinction so that an organism can make context-appropriate decisions. Future studies will examine whether it is possible to exploit these separable processes to inhibit contextual encoding and facilitate extinction to prevent renewal of cocaine seeking.

\section{References}

Baeg EH, Jackson ME, Jedema HP, Bradberry CW (2009) Orbitofrontal and anterior cingulate cortex neurons selectively process cocaine-associated environmental cues in the rhesus monkey. J Neurosci 29:11619-11627. CrossRef Medline

Botvinick MM (2007) Conflict monitoring and decision making: reconciling two perspectives on anterior cingulate function. Cogn Affect Behav Neurosci 7:356-366. CrossRef Medline

Bouton ME (2004) Context and behavioral processes in extinction. Learn Mem 11:485-494. CrossRef Medline

Bouton ME, Westbrook RF, Corcoran KA, Maren S (2006) Contextual and temporal modulation of extinction: behavioral and biological mechanisms. Biol Psychiatry 60:352-360. CrossRef Medline

Bouton ME, Winterbauer NE, Todd TP (2012) Relapse processes after the extinction of instrumental learning: renewal, resurgence, and reacquisition. Behav Processes 90:130-141. CrossRef Medline

Bryden DW, Johnson EE, Tobia SC, Kashtelyan V, Roesch MR (2011) Attention for learning signals in anterior cingulate cortex. J Neurosci 31: 18266-18274. CrossRef Medline

Corcoran KA, Desmond TJ, Frey KA, Maren S (2005) Hippocampal inactivation disrupts the acquisition and contextual encoding of fear extinction. J Neurosci 25:8978-8987. CrossRef Medline

Crombag HS, Bossert JM, Koya E, Shaham Y (2008) Review. Contextinduced relapse to drug seeking: a review. Philos Trans R Soc Lond B Biol Sci 363:3233-3243. CrossRef Medline

Hobin JA, Ji J, Maren S (2006) Ventral hippocampal muscimol disrupts context-specific fear memory retrieval after extinction in rats. Hippocampus 16:174-182. CrossRef Medline

Ji J, Maren S (2005) Electrolytic lesions of the dorsal hippocampus disrupt renewal of conditional fear after extinction. Learn Mem 12:270-276. CrossRef Medline

Ji J, Maren S (2008a) Differential roles for hippocampal areas CA1 and CA3 in the contextual encoding and retrieval of extinguished fear. Learn Mem 15:244-251. CrossRef Medline

Ji J, Maren S (2008b) Lesions of the entorhinal cortex or fornix disrupt the context-dependence of fear extinction in rats. Behav Brain Res 194:201206. CrossRef Medline

Kennerley SW, Behrens TE, Wallis JD (2011) Double dissociation of value computations in orbitofrontal and anterior cingulate neurons. Nat Neurosci 14:1581-1589. CrossRef Medline

Klavir O, Genud-Gabai R, Paz R (2012) Low-frequency stimulation depresses the primate anterior-cingulate-cortex and prevents spontaneous recovery of aversive memories. J Neurosci 32:8589-8597. CrossRef Medline

Knackstedt LA, Moussawi K, Lalumiere R, Schwendt M, Klugmann M, Kalivas PW (2010) Extinction training after cocaine self-administration in- 
duces glutamatergic plasticity to inhibit cocaine seeking. J Neurosci 30: 7984-7992. CrossRef Medline

Lang S, Kroll A, Lipinski SJ, Wessa M, Ridder S, Christmann C, Schad LR, Flor $\mathrm{H}$ (2009) Context conditioning and extinction in humans: differential contribution of the hippocampus, amygdala and prefrontal cortex. Eur J Neurosci 29:823-832. CrossRef Medline

Myers KM, Carlezon WA Jr (2012) D-cycloserine effects on extinction of conditioned responses to drug-related cues. Biol Psychiatry 71:947-955. CrossRef Medline

Olausson P, Jentsch JD, Taylor JR (2004) Repeated nicotine exposure enhances responding with conditioned reinforcement. Psychopharmacology 173:98-104. CrossRef Medline

Paxinos G, Watson C (2005) The rat brain in stereotaxic coordinates. Amsterdam: Elsevier.

Peters J, Dieppa-Perea LM, Melendez LM, Quirk GJ (2010) Induction of fear extinction with hippocampal-infralimbic BDNF. Science 328: 1288-1290. CrossRef Medline

Price KL, Baker NL, McRae-Clark AL, Saladin ME, Desantis SM, Santa Ana EJ, Brady KT (2013) A randomized, placebo-controlled laboratory study of the effects of D-cycloserine on craving in cocaine-dependent individuals. Psychopharmacology 226:739-746. CrossRef Medline

Ressler KJ, Rothbaum BO, Tannenbaum L, Anderson P, Graap K, Zimand E, Hodges L, Davis M (2004) Cognitive enhancers as adjuncts to psychotherapy: use of D-cycloserine in phobic individuals to facilitate extinction of fear. Arch Gen Psychiatry 61:1136-1144. CrossRef Medline

Roesch MR, Esber GR, Li J, Daw ND, Schoenbaum G (2012) Surprise! Neural correlates of Pearce-Hall and Rescorla-Wagner coexist within the brain. Eur J Neurosci 35:1190-1200. CrossRef Medline

Rushworth MF, Noonan MP, Boorman ED, Walton ME, Behrens TE (2011) Frontal cortex and reward-guided learning and decision-making. Neuron 70:1054-1069. CrossRef Medline

Taylor JR, Robbins TW (1984) Enhanced behavioural control by conditioned reinforcers following microinjections of $\mathrm{d}$-amphetamine into the nucleus accumbens. Psychopharmacology 84:405-412. CrossRef Medline
Taylor JR, Robbins TW (1986) 6-Hydroxydopamine lesions of the nucleus accumbens, but not of the caudate nucleus, attenuate enhanced responding with reward-related stimuli produced by intra-accumbens d-amphetamine. Psychopharmacology 90:390-397. Medline

Taylor JR, Olausson P, Quinn JJ, Torregrossa MM (2009) Targeting extinction and reconsolidation mechanisms to combat the impact of drug cues on addiction. Neuropharmacology 56 [Suppl 1]:186-195. CrossRef Medline

Torregrossa MM, Kalivas PW (2008) Neurotensin in the ventral pallidum increases extracellular gamma-aminobutyric acid and differentially affects cue- and cocaine-primed reinstatement. J Pharmacol Exp Ther 325: 556-566. CrossRef Medline

Torregrossa MM, Taylor JR (2013) Learning to forget: manipulating extinction and reconsolidation processes to treat addiction. Psychopharmacology 226:659-672. CrossRef Medline

Torregrossa MM, Sanchez H, Taylor JR (2010) D-Cycloserine reduces the context specificity of pavlovian extinction of cocaine cues through actions in the nucleus accumbens. J Neurosci 30:10526-10533. CrossRef Medline

Totah NK, Kim YB, Homayoun H, Moghaddam B (2009) Anterior cingulate neurons represent errors and preparatory attention within the same behavioral sequence. J Neurosci 29:6418-6426. CrossRef Medline

Walton ME, Mars RB (2007) Probing human and monkey anterior cingulate cortex in variable environments. Cogn Affect Behav Neurosci 7:413422. CrossRef Medline

Walton ME, Croxson PL, Behrens TEJ, Kennerley SW, Rushworth MFS (2007) Adaptive decision making and value in the anterior cingulate cortex. Neuroimage 36 [Suppl 2]:T142-T154. Medline

Watson BJ, Wilson S, Griffin L, Kalk NJ, Taylor LG, Munafò MR, LingfordHughes AR, Nutt DJ (2011) A pilot study of the effectiveness of D-cycloserine during cue-exposure therapy in abstinent alcoholdependent subjects. Psychopharmacology 216:121-129. CrossRef Medline

Westbrook RF, Good AJ, Kiernan MJ (1997) Microinjection of morphine into the nucleus accumbens impairs contextual learning in rats. Behav Neurosci 111:996-1013. CrossRef Medline 